\title{
Metformin Prevents Nonunion after Three-Cannulated-Screw Fixation in Displaced Femoral Neck Fractures: A Retrospective Study
}

\author{
Xiao-zhong Zhu and Xian-you Zheng \\ Department of Orthopaedic Surgery, Shanghai Jiaotong University Affiliated Sixth People's Hospital, Shanghai 200233, China \\ Correspondence should be addressed to Xian-you Zheng; shzhengxianyou@163.com
}

Received 10 August 2016; Revised 7 October 2016; Accepted 24 October 2016

Academic Editor: Frankie Leung

Copyright (C) 2016 X.-z. Zhu and X.-y. Zheng. This is an open access article distributed under the Creative Commons Attribution License, which permits unrestricted use, distribution, and reproduction in any medium, provided the original work is properly cited.

\begin{abstract}
Patients aged from 40 to 60 with displaced fractures of the femoral neck (Garden III or IV) who received fixation with three cannulated screws from January 2005 to December 2012 were evaluated retrospectively for the development of nonunion. Plasma $\mathrm{HbAlC}$, a marker for long-term plasma glucose level, anti-T2DM medication, and other potential risk factors were recorded for the purpose of this study. There were no differences between the union and nonunion groups with respect to age, gender, Garden classification, Pauwel's angle, reduction quality, and T2DM presence. There were significant differences in reduction quality and preoperative plasma $\mathrm{HbAlC}$ level between patients with and those without union. The odds ratio (OR) for fracture nonunion was 2.659 (95\% confidence interval [CI], 1.530-4.620) in subjects with anatomical reduction compared with those without anatomical reduction, 4.797 ( $95 \% \mathrm{CI}, 1.371-16.778$ ), in subjects with poor blood glucose control (HbA1C > 10\%). The metformin usage showed a preventive effect on nonunion development (OR: 0.193 and CI: $0.060-0.616)$. The nonunion rate of metformin group $(6.7 \%, 6 / 89)$ was even much lower than that of patients without T2DM $(17.4 \%, 80 / 460)$
\end{abstract}

\section{Background}

The nonunion is one of the common complications after femoral neck fractures. Reported incidence of nonunion after three-cannulated-screw fixation ranged from $13.3 \%$ to $21.8 \%$ and many risk factors including impropriate crew fixation configuration, displaced fracture, and poor reduction have been revealed [1-3].

Type 2 diabetes mellitus (T2DM) affects 382 million individuals across the world and its prevalence is steadily rising [4]. T2DM leads to the fragility of bones by affecting the bone metabolism [5]. Emerging evidences have indicated T2DM as an independent risk factor for fractures $[6,7]$. Hence, management of fractures in T2DM patients has become an increasingly important topic in orthopedic practice and research.

T2DM is characterized by a series of micro- and macrovascular complications caused by insulin resistance [8]. In addition, insulin resistance restrains the reestablishment of vascularization, thereby impairing the wound healing $[9,10]$.
Previous study has demonstrated that T2DM is associated with impaired fracture healing including delayed union and nonunion [11]. The nonunion after foot and ankle surgery is more common in patients with T2DM [12]. However, the influence of T2DM on femoral neck nonunion is not clear.

\section{Methods}

All patients with fresh displaced femoral neck fractures (Garden III or IV) who received surgical fixation with three cannulated screws from January 2005 to December 2012 in our hospital were assessed for eligibility for study inclusion. The following inclusion criteria were applied: age from 40 to 60 , surgery time within 30 days after the injury, and having glycated hemoglobin (HbAlC) detection before surgery. Patients with any of the following criteria were excluded: T1DM, pathological fracture, additional fracture in the ipsilateral lower limb or pelvis, and loss of follow-up within 24 months after surgery. A total of 601 patients were included in our analysis. Data from the included patients 
TABLE 1: Demographic information and characteristics of the fractures.

\begin{tabular}{|c|c|c|c|c|}
\hline & Total $(n=601)$ & Union $(n=503)$ & Nonunion $(n=98)$ & $P$ value \\
\hline Male & 337 & 290 & 47 & \multirow{2}{*}{0.077} \\
\hline Female & 264 & 213 & 51 & \\
\hline $\mathrm{Age}^{\dagger}$ & $50.2 \pm 5.1$ & $50.2 \pm 5.2$ & $49.8 \pm 5.1$ & 0.471 \\
\hline $\mathrm{BMI}^{\dagger}$ & $23.1 \pm 2.4$ & $23.1 \pm 2.4$ & $22.9 \pm 2.2$ & 0.428 \\
\hline \multicolumn{5}{|c|}{ Garden classification ${ }^{*}$} \\
\hline Stage III & 350 & 298 & 52 & \multirow{2}{*}{0.257} \\
\hline Stage IV & 251 & 205 & 46 & \\
\hline \multicolumn{5}{|c|}{ Pauwel's classification* ${ }^{*}$} \\
\hline Stage I & 55 & 44 & 11 & \multirow{3}{*}{0.493} \\
\hline Stage II & 368 & 313 & 55 & \\
\hline Stage III & 178 & 146 & 32 & \\
\hline \multicolumn{5}{|l|}{ Reduction quality ${ }^{*}$} \\
\hline Anatomical & 519 & 446 & 73 & \multirow{2}{*}{0.0002} \\
\hline Nonanatomical & 82 & 57 & 25 & \\
\hline \multicolumn{5}{|l|}{ T2DM* } \\
\hline- & 460 & 380 & 80 & \multirow{2}{*}{0.193} \\
\hline+ & 141 & 123 & 18 & \\
\hline \multicolumn{5}{|l|}{$\mathrm{HbAlC}^{\S}$} \\
\hline$<6 \%$ & 475 & 393 & 82 & \multirow{3}{*}{0.041} \\
\hline $6-10 \%$ & 100 & 91 & 9 & \\
\hline$>10 \%$ & 26 & 19 & 7 & \\
\hline \multicolumn{5}{|l|}{ Metformin ${ }^{*}$} \\
\hline- & 512 & 420 & 92 & \multirow{2}{*}{0.008} \\
\hline+ & 89 & 83 & 6 & \\
\hline \multicolumn{5}{|l|}{ Insulin* } \\
\hline- & 526 & 435 & 91 & \multirow{2}{*}{0.081} \\
\hline+ & 75 & 68 & 7 & \\
\hline \multicolumn{5}{|c|}{ Alpha-glucosidase inhibitor ${ }^{\S}$} \\
\hline- & 573 & 477 & 96 & \multirow{2}{*}{0.179} \\
\hline+ & 28 & 26 & 2 & \\
\hline \multicolumn{5}{|l|}{ Secretagogue* } \\
\hline- & 549 & 458 & 91 & \multirow{2}{*}{0.561} \\
\hline+ & 52 & 45 & 7 & \\
\hline
\end{tabular}

${ }^{*} P$ values are based on the chi-square test. ${ }^{\dagger} P$ values are based on Student's $t$-test. ${ }^{\S} P$ values are based on the Fisher exact test.

were analyzed retrospectively. The following preoperative variables were recorded: age, gender, body mass index (BMI), and glycated hemoglobin (HbA1C). Demographic information and characteristics of the fractures before surgery was summarized in Table 1. T2DM-related medication after surgery was recorded before analysis. The T2DM-related medications for each patient were summarized in Supplementary Table 1, in Supplementary Material available online at http://dx.doi.org/10.1155/2016/5682541. According to previous studies, nonunion was defined as a loss of reduction or fixation after six weeks or radiological absence of union at one year $[1,13]$.

Statistical analysis was performed using SPSS 22.0 software. A $P$ value of $<0.05$ was considered to be statistically significant. Fisher's exact test was used to compare nonunion rates between the groups. Data were presented as mean \pm standard deviation.

\section{Results}

All the potential risk factors, including Garden classification, Pauwel's angle, reduction quality, T2DM presence, preoperative plasma HbA1C level, and T2DM-related medications, were considered as covariates, respectively. The development of nonunion was considered as dependent variable. The results of multivariate binary logistic regression analysis were shown in Table 2. Reduction quality instead of the fractures characteristics was associated with the occurrence of nonunion, which is consistent with previous studies [14]. The presence of T2DM did not significantly increase or decrease the nonunion rate in this study. The OR for fracture nonunion was 4.797 (95\% CI, 1.371-16.778) in subjects with poor blood glucose control ( $\mathrm{HbA1C}>10 \%)$.

We also analyzed whether T2DM-related medications were associated with the nonunion rate. The metformin 
TABLE 2: Results of multivariate binary logistic regression analysis.

\begin{tabular}{|c|c|c|c|}
\hline & $P$ value & OR & $95 \% \mathrm{CI}$ \\
\hline Garden Stage IV (reference: Garden Stage III) & 0.284 & 1.280 & $0.814-2.013$ \\
\hline Pauwel's Stage II (reference: Pauwel's Stage I) & 0.223 & 0.630 & $0.300-1.323$ \\
\hline Pauwel's Stage III (reference: Pauwel's Stage I) & 0.443 & 1.219 & $0.735-2.022$ \\
\hline Reduction quality (reference: anatomical) ${ }^{*}$ & 0.001 & 2.659 & $1.530-4.620$ \\
\hline T2DM (+) & 0.241 & 3.030 & $0.474-19.356$ \\
\hline HbAlC 6\%-10\% (reference: $\mathrm{HbA1C}<6 \%$ ) & 0.385 & 2.341 & $0.343-15.984$ \\
\hline $\mathrm{HbAlC}>10 \%$ (reference: $\mathrm{HbAlC}<6 \%)^{*}$ & 0.014 & 4.797 & $1.371-16.778$ \\
\hline $\operatorname{Metformin}(+)^{*}$ & 0.005 & 0.193 & $0.060-0.616$ \\
\hline Insulin $(+)$ & 0.101 & 0.386 & $0.124-1.204$ \\
\hline Alpha-glucosidase inhibitor $(+)$ & 0.363 & 0.459 & $0.086-2.456$ \\
\hline Secretagogue $(+)$ & 0.875 & 1.094 & $0.356-3.362$ \\
\hline
\end{tabular}

$\mathrm{OR}=$ odds ratio $\mathrm{CI}=$ confidence interval. ${ }^{*} P<0.05$.

usage showed a preventive effect on nonunion development (OR: 0.183 and CI: 0.058-0.577) while other T2DM-related medications showed no such effect. There was no significant difference in preoperative plasma HbA1C level between T2DM patients with and without metformin usage $(P>$ 0.05 , Student's $t$-test) suggesting the prevention of metformin might be irrelevant to the anti-T2DM effect and improved T2DM control. Supporting our notion, the nonunion rate of metformin group $(6.7 \%, 6 / 89)$ was even much lower than that of patients without T2DM $(17.4 \%, 80 / 460)$.

\section{Discussion}

In this study, we found that the T2DM presence was not an independent risk factor for fracture nonunion. Increased risks for the development of fracture nonunion after internal fixation of femoral neck fractures were only found in patients with poor blood glucose control. Our observation suggested that the inhibitory effect of T2DM on fracture healing might be negated after ideal control of plasma glucose level.

Metformin has been shown to have osteogenesis-promoting effects in vitro [15]. In addition, metformin inhibits osteoclast differentiation by reducing receptor activator of nuclear factor $\kappa \mathrm{B}$ ligand expression [16]. Theoretically, metformin could promote bone formation and thus facilitate fracture healing. However, the studies based on animal models have shown controversial effects of metformin on fracture healing $[17,18]$. Our study supported the notion that metformin might prevent the nonunion after femoral neck fracture by providing clinical evidences.

It is possible that the preventive effect of metformin was associated with its glucose-lowering effect. The preoperative plasma HbA1C levels between T2DM patients with and those without metformin usage had no significant difference suggesting the preventive effect of metformin might be independent of plasma glucose control. In addition, other anti-T2DM medications showed no preventive effect on nonunion. Most importantly, the nonunion rate of metformin group was even significantly lower compared to patients without T2DM.

Since the side effects of metformin are mild, patients with femoral fractures might benefit from usage of metformin even without T2DM presence. Future perspective studies and clinical trials might further confirm our observation.

We also found that fracture without anatomical reduction was a significant risk factor for nonunion in displaced femoral neck fractures, whereas fracture angle was not. A more horizontal fracture line was associated with internal fixation failure in clinical and mechanical studies [19]. However, we did not find correlation between Pauwel's classification and the development of nonunion.

This study has some limitations. The T2DM-related medications were recorded before analysis by contacting participants and thereby the recall bias could not be entirely avoided. However, the T2DM-related medication is generally applied in long term and the alteration on antidiabetic drugs in T2DM patients is uncommon. Hence, the recall bias might be mild and main conclusion of this study is not affected. Clearly, a perspective study is required for further confirmation on our findings.

\section{Conclusion}

Metformin can decrease the nonunion rate of femoral neck fractures independent of its anti-T2DM effect.

\section{Abbreviations \\ T2DM: Type 2 diabetes mellitus \\ OR: Odds ratio \\ CI: Confidence interval \\ HbA1C: Hemoglobin A1C.}

\section{Additional Points}

Availability of Data and Materials. The dataset supporting the conclusions presented in this article is available on request from the corresponding author.

\section{Ethical Approval}

This study was approved by the Ethics Committee of Shanghai Jiaotong University Affiliated Sixth People's Hospital. 


\section{Consent}

Informed consents were obtained from all donors included in this study in accordance with the World Medical Association Declaration of Helsinki.

\section{Competing Interests}

All authors declare no competing interests.

\section{Authors' Contributions}

Xian-you Zheng was responsible for study design and manuscript writing. Xiao-zhong Zhu was responsible for data collection and analysis.

\section{Acknowledgments}

The authors acknowledge the contribution of all patients included in this study. They are grateful for the constructive suggestions from Dr. Hongyi Zhu. This study was supported by National Natural Science Foundation of China (81371965), Shanghai Municipal Education Commission-Gaofeng Clinical Medicine Grant (20161429), and Shanghai Pujiang Program (16PJD035).

\section{References}

[1] J.-J. Yang, L.-C. Lin, K.-H. Chao et al., "Risk factors for nonunion in patients with intracapsular femoral neck fractures treated with three cannulated screws placed in either a triangle or an inverted triangle configuration," The Journal of Bone \& Joint Surgery-American Volume, vol. 95, no. 1, pp. 61-69, 2013.

[2] T. P. Araujo, T. M. Guimaraes, F. B. Andrade-Silva, K. E. Kojima, and S. Silva Jdos, "Influence of time to surgery on the incidence of complications in femoral neck fracture treated with cannulated screws," Injury, vol. 45, supplement 5, pp. S36-S39, 2014.

[3] S. Kumar, A. Bharti, A. Rawat, V. Kumar, and S. Avasthi, "Comparative study of fresh femoral neck fractures managed by multiple cancellous screws with and without fibular graft in young adults," Journal of Clinical Orthopaedics and Trauma, vol. 6, no. 1, pp. 6-11, 2015.

[4] A. D. Dede, S. Tournis, I. Dontas, and G. Trovas, "Type 2 diabetes mellitus and fracture risk," Metabolism, vol. 63, no. 12, pp. 1480-1490, 2014.

[5] T. Yamaguchi and T. Sugimoto, "Bone metabolism and fracture risk in type 2 diabetes mellitus [Review]," Endocrine Journal, vol. 58, no. 8, pp. 613-624, 2011.

[6] E. A. C. de Waard, T. A. C. M. van Geel, H. H. C. M. Savelberg, A. Koster, P. P. M. M. Geusens, and J. P. W. van den Bergh, "Increased fracture risk in patients with type 2 diabetes mellitus: an overview of the underlying mechanisms and the usefulness of imaging modalities and fracture risk assessment tools," Maturitas, vol. 79, no. 3, pp. 265-274, 2014.

[7] M. Yamamoto, "Insights into bone fragility in diabetes: the crucial role of bone quality on skeletal strength," Endocrine Journal, vol. 62, no. 4, pp. 299-308, 2015.
[8] Q.-A. Altaf, A. H. Barnett, and A. A. Tahrani, "Novel therapeutics for type 2 diabetes: insulin resistance," Diabetes, Obesity \& Metabolism, vol. 17, no. 4, pp. 319-334, 2015.

[9] Y.-C. Lim, M. P. Bhatt, M.-H. Kwon et al., "Proinsulin C-Peptide prevents impaired wound healing by activating angiogenesis in diabetes," The Journal of Investigative Dermatology, vol. 135, no. 1, pp. 269-278, 2015.

[10] M. Hrynyk and R. J. Neufeld, "Insulin and wound healing," Burns, vol. 40, no. 8, pp. 1433-1446, 2014.

[11] R. K. Hernandez, T. P. Do, C. W. Critchlow, R. E. Dent, and S. S. Jick, "Patient-related risk factors for fracture-healing complications in the United Kingdom General Practice Research Database," Acta Orthopaedica, vol. 83, no. 6, pp. 653-660, 2012.

[12] N. Shibuya, J. M. Humphers, B. L. Fluhman, and D. C. Jupiter, "Factors associated with nonunion, delayed union, and malunion in foot and ankle surgery in diabetic patients," The Journal of Foot and Ankle Surgery, vol. 52, no. 2, pp. 207-211, 2013.

[13] A. Upadhyay, P. Jain, P. Mishra, L. Maini, V. K. Gautum, and B. K. Dhaon, "Delayed internal fixation of fractures of the neck of the femur in young adults. A prospective, randomised study comparing closed and open reduction," The Journal of Bone and Joint Surgery - British Volume, vol. 86, no. 7, pp. 1035-1040, 2004.

[14] Y.-S. Gao, Z.-S. Ai, Z.-H. Zhu, X.-W. Yu, and C.-Q. Zhang, "Injury-to-surgery interval does not affect postfracture osteonecrosis of the femoral head in young adults: a systematic review," European Journal of Orthopaedic Surgery and Traumatology, vol. 23, no. 2, pp. 203-209, 2013.

[15] A. M. Cortizo, C. Sedlinsky, A. D. McCarthy, A. Blanco, and L. Schurman, "Osteogenic actions of the anti-diabetic drug metformin on osteoblasts in culture," European Journal of Pharmacology, vol. 536, no. 1-2, pp. 38-46, 2006.

[16] Q.-G. Mai, Z.-M. Zhang, S. Xu et al., "Metformin stimulates osteoprotegerin and reduces RANKL expression in osteoblasts and ovariectomized rats," Journal of Cellular Biochemistry, vol. 112, no. 10, pp. 2902-2909, 2011.

[17] J. Jeyabalan, B. Viollet, P. Smitham et al., "The anti-diabetic drug metformin does not affect bone mass in vivo or fracture healing," Osteoporosis International, vol. 24, no. 10, pp. 26592670, 2013.

[18] J. La Fontaine, C. Chen, N. Hunt, E. Jude, and L. Lavery, “Type 2 diabetes and metformin influence on fracture healing in an experimental rat model," The Journal of Foot and Ankle Surgery, vol. 55, no. 5, pp. 955-960, 2016.

[19] C. J. Stankewich, J. Chapman, R. Muthusamy et al., "Relationship of mechanical factors to the strength of proximal femur fractures fixed with cancellous screws," Journal of Orthopaedic Trauma, vol. 10, no. 4, pp. 248-257, 1996. 


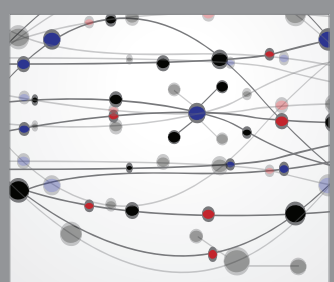

The Scientific World Journal
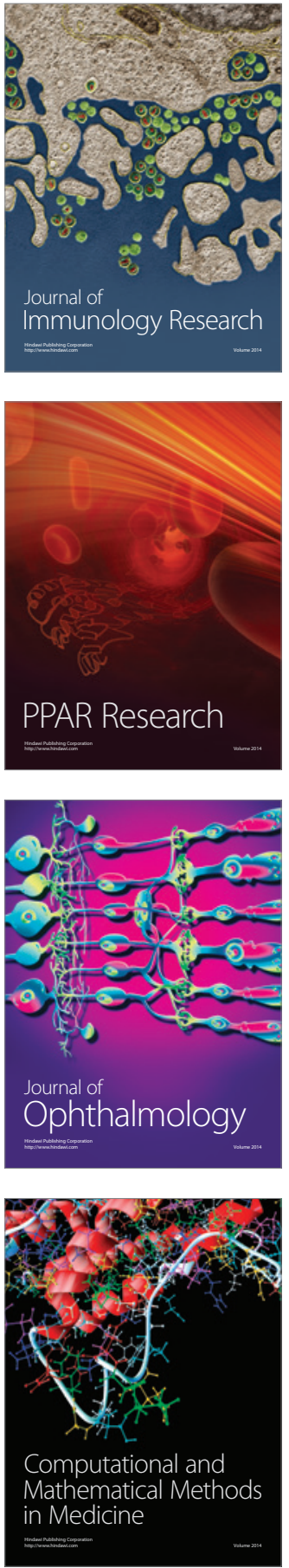

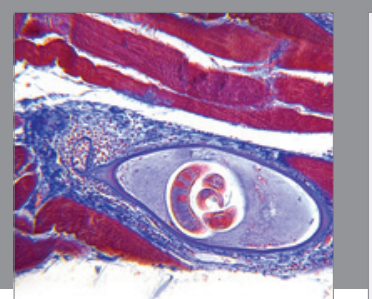

Gastroenterology Research and Practice

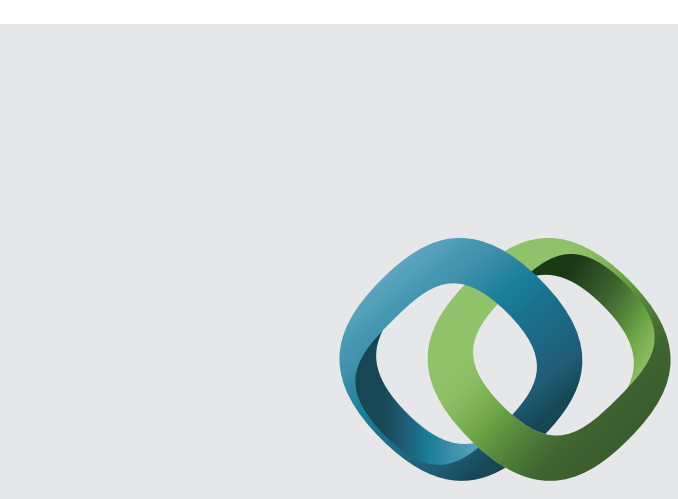

\section{Hindawi}

Submit your manuscripts at

http://www.hindawi.com
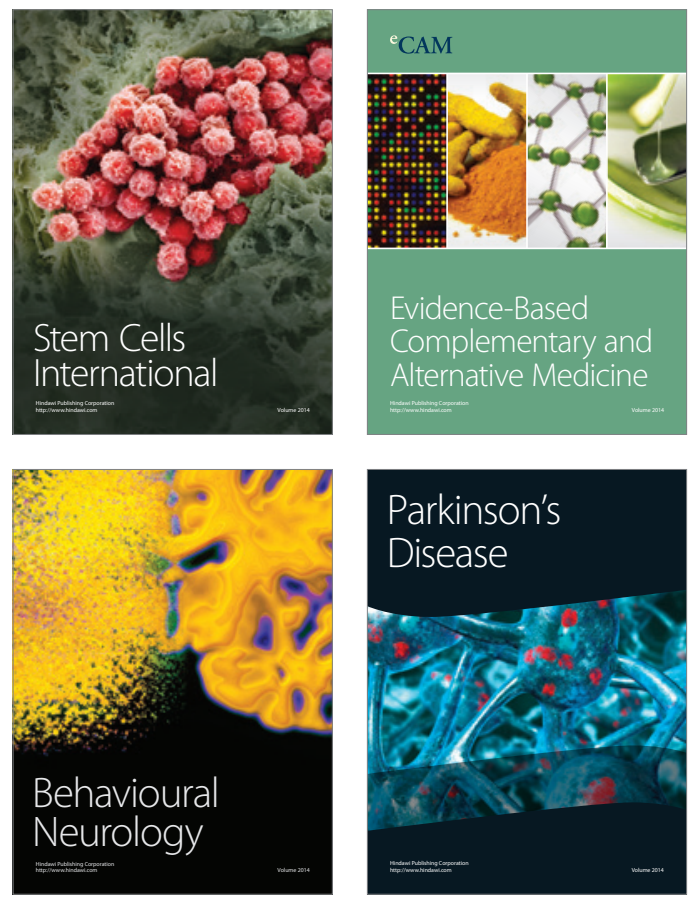
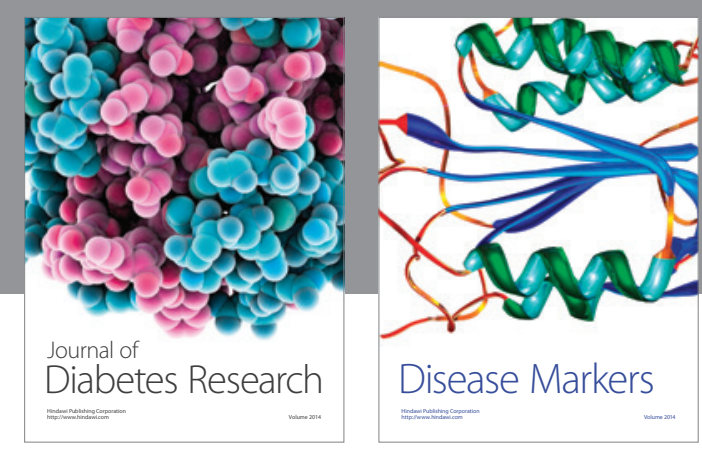

Disease Markers
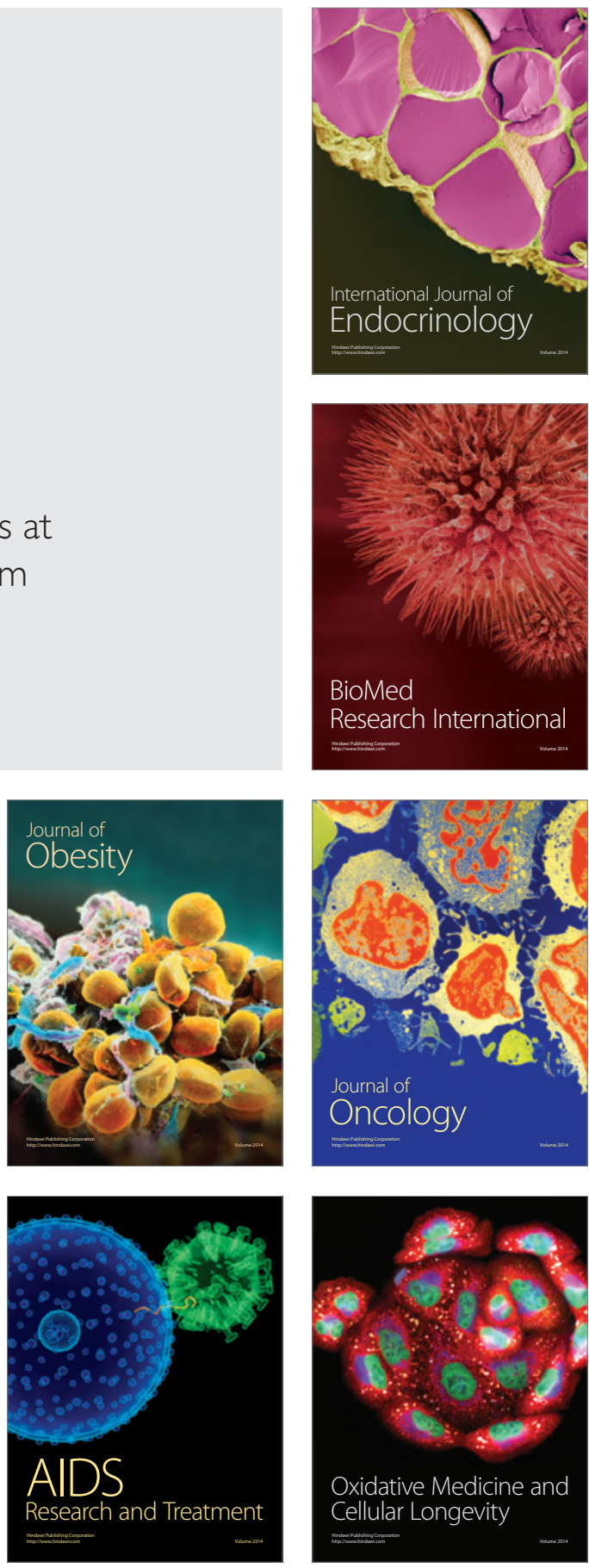